\title{
Dual-Frequency Optical Pumping for Spin-Polarizing a Lithium Atomic Beam
}

\author{
G. Baum, C.D.Caldwell ${ }^{\star}$, and W.Schröder \\ Fakultät für Physik, Universität, D-4800 Bielefeld, Fed. Rep. Germany
}

Received 20 August 1979/Accepted 23 October 1979

\begin{abstract}
A lithium-6 atomic beam is spin-polarized by means of optical pumping with a single-mode dye laser operating on the resonance transition. Simultaneous pumping of both hyperfine substates is achieved by frequency-splitting the laser light with an acousto-optic modulator. A polarization dependent signal, obtained by probing the optical activity of the beam with linearly polarized light, is utilized in a microprocessor-controlled laser stabilization scheme. The polarization is analyzed with a sextupole magnet and its overall value is 0.70 for an intensity of $1 \times 10^{14}$ atoms $\mathrm{s}^{-1}$. By reversing the sense of circular polarization of the pumping light the atomic beam polarization is easily reversed in direction.
\end{abstract}

PACS: $32.90+a, 42.60-v, 35.80+s$

In recent years interest has grown in investigating angular-resolved effects in crossed-beam scattering. For a system of two spin-1/2 particles it is possible to measure both the singlet and triplet scattering cross sections and their relative phase provided two polarization states of the incoming or outgoing particles are known (see, for instance, the articles by Kleinpoppen [1] and Wainwright et al. [2]). With the intention of performing such an experiment using electrons and lithium atoms [3], we have studied the spinpolarization of lithium atomic beams.

For such an experiment it is desirable to have a polarization close to unity which, in addition, should be easily reversible in direction. In the low magnetic fields in which the scattering must take place it is impossible to achieve these requirements with conventional methods, i.e. Stern-Gerlach or sextupole magnets. Therefore, we have decided to use the method of optical pumping ${ }^{1}$. This should allow us to obtain a high polarization in the atomic beam, which is easily reversible by reversing the sense of circular polarization of the radiation.

\footnotetext{
* Present address: Physics Department, Yale University, New Haven, CT06520, USA.

1 An excellent summary of optical pumping has been given by
} Happer [4].

\section{Method}

The optical pumping method is based on the absorption of polarized, unidirectional photons by the different Zeeman states of atoms. If we look at the diagram in Fig. 1, we see that circularly polarized resonance radiation $\left(\sigma^{+}\right)$will cause transitions from ground state $M_{F}$ levels to an $M_{F}$, level one unit larger (heavy lines). (The following discussion will be given for $\sigma^{+}$pumping; the same considerations hold for $\sigma^{-}$ pumping with the helicity reversed). Upon reaching these excited states, the system then decays back to all Zeeman levels of the ground state via spontaneous emission $\left(\Delta M_{F}=0, \pm 1\right)$ or is forced back into the original level by induced emission. If the exciting light is intense enough, the atoms will be reexcited after decaying, and the process repeats itself. We note that absorption of the $\sigma^{+}$light followed by spontaneous emission tends to shift the atoms into Zeeman states with higher $M_{F}$ values. If the process is repeated frequently enough, all atoms will accumulate in the Zeeman level on the right, and the resulting ensemble will be spin-polarized.

In order to achieve high polarizations, the exciting light must have a high intensity, as the rate of absorption is thereby increased. Yet, as the intensity of the light source is increased, the decay due to induced 


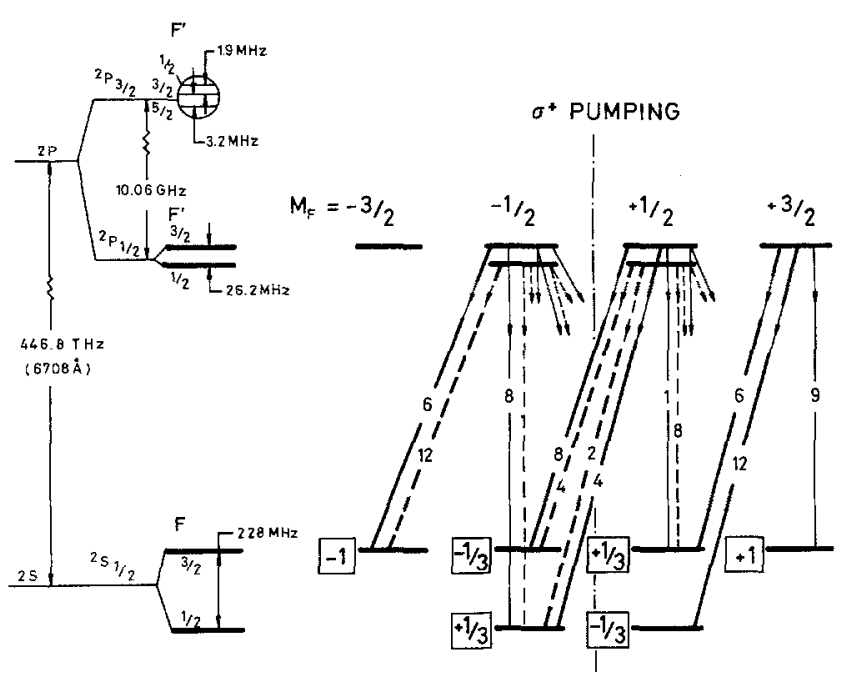

Fig. 1. Energy levels and pumping diagram for lithium-6. Solid lines in the pumping diagram connect to the excited $F^{\prime}=3 / 2$ state, dashed lines to the $F^{\prime}=1 / 2$ state. Heavy lines refer to transitions induced by $\sigma^{+} \operatorname{light}\left(A M_{F}=+1\right)$. The numbers at each line denote the relative strengths of the transition. The spontaneous decay transition $\left(\Delta M_{F}=0, \pm 1\right)$ are indicated by the downward pointing arrows. Not all of these transitions are fully shown, but can be obtained from the mirror images with respect to the center line. The electronic polarization of each ground-state sublevel is given in the boxes

emission increases as well. This induced emission cannot produce a change in polarization, however, as it always returns the system to its original Zeeman level. If the time which the atom spends in the light beam is much longer than the spontaneous lifetime of the excited state, the induced emission will have no net effect on the ultimate polarization which can be achieved. Thus, while the induced processes do not hurt, they also do not help. Therefore, to prevent wasting valuable intensity, we spread the light over a longer path in the beam. While the photon flux remains constant, its effectiveness for spontaneous processes is enhanced.

The first optical pumping experiments on alkali vapors were performed with discharge lamps, however, because of its high spectral density, the laser is more suited for the process especially for experiments on atomic beams. One advantage of the discharge lamp, though is that it pumps both of the hyperfine components simultaneously. This is harder to do with a laser, as the narrow bandwidth allows one to resolve the ground state hyperfine structure completely. That both components must be pumped to achieve maximum polarization ${ }^{2}$ can be seen from Fig. 1, which

\footnotetext{
${ }^{2}$ It is possible to achieve high polarization by pumping only the $F=3 / 2$ component if the pumping is carried out over the ${ }^{2} P_{3 / 2}$, $F^{\prime}=5 / 2$ excited state. Yet, as the $F=1 / 2$ ground level is not affected by this process, the maximum polarization obtainable is 0.67 and it requires resolving the hyperfine components in the excited state.
}

gives the spin-polarization in each of the Zeeman levels. For $\sigma^{+}$pumping maximum polarization corresponds to all atoms being in the $F=3 / 2, M_{F}=+3 / 2$ state, and it is especially crucial that no atoms remain in the $F=1 / 2, M_{F}=+1 / 2$ state, as this level has a spinpolarization which is negative.

There have been several suggestions as to how to excite both hyperfine components using only one laser. One is to generate both frequencies within the laser itself $[5,6]$. Another is to take advantage of the Doppler shift in the atomic beam [7-9]. The single-frequency laser beam is split into two or more beams and allowed to intersect the atomic beam at different angles. These angles are so chosen that the relative Doppler shift corresponds to the hyperfine splitting. While this technique is quite effective, it does have the drawback that it is highly velocity selective.

We have employed a different means of producing the two required beams. It utilizes the acousto-optical effect $[10]$ to split the single laser beam from the dye laser into two beams, one with the original frequency and the other shifted by plus or minus the oscillation frequency of the crystal. This method is limited to frequency shifts up to approximately $400 \mathrm{MHz}$ due to the availability of transducers. For this reason we choose to use lithium- 6 because of its smaller ground state hyperfine splitting $(228 \mathrm{MHz})$ as compared to that of lithium-7 (804 MHz).

Since both ground-state hyperfine levels are affected by the laser, the pump process can be carried out over any of the excited-state fine of hyperfine levels. However, Franzen and Emslie [11] showed by calculations that a higher degree of polarization could be achieved if only the ${ }^{2} S_{1 / 2} \rightarrow{ }^{2} P_{1 / 2}$ fine structure transition is used for pumping. The choice of which hyperfine level of the excited state to use is not crucial to the results of the experiment as long as the pumping is not carried out solely over levels with $F^{\prime} \leqq|1 / 2-I|$.

\section{Experimental Arrangement}

The lithium atomic beam is produced from an effusive oven, the details of which have been described elsewhere [12]. The beam is collimated (collimation ratio 100 ) to a diameter of $0.5 \mathrm{~cm}$ at the point of interaction with the laser beam and is monitored further downstream with a Langmuir-Taylor detector using an oxidized tungsten wire. We operate the oven at $750^{\circ} \mathrm{C}$, producing a beam intensity of approximately $1 \times 10^{14}$ atoms $/ \mathrm{s}$. The lithium has a stated isotopic content of $95 \%$ lithium- 6 and $5 \%$ lithium-7. Through additional heating of the orifice to roughly $850^{\circ} \mathrm{C}$ we reduce the content of $\mathrm{Li}_{2}$ which is contained in the vapor. On the basis of thermodynamic calculations we 


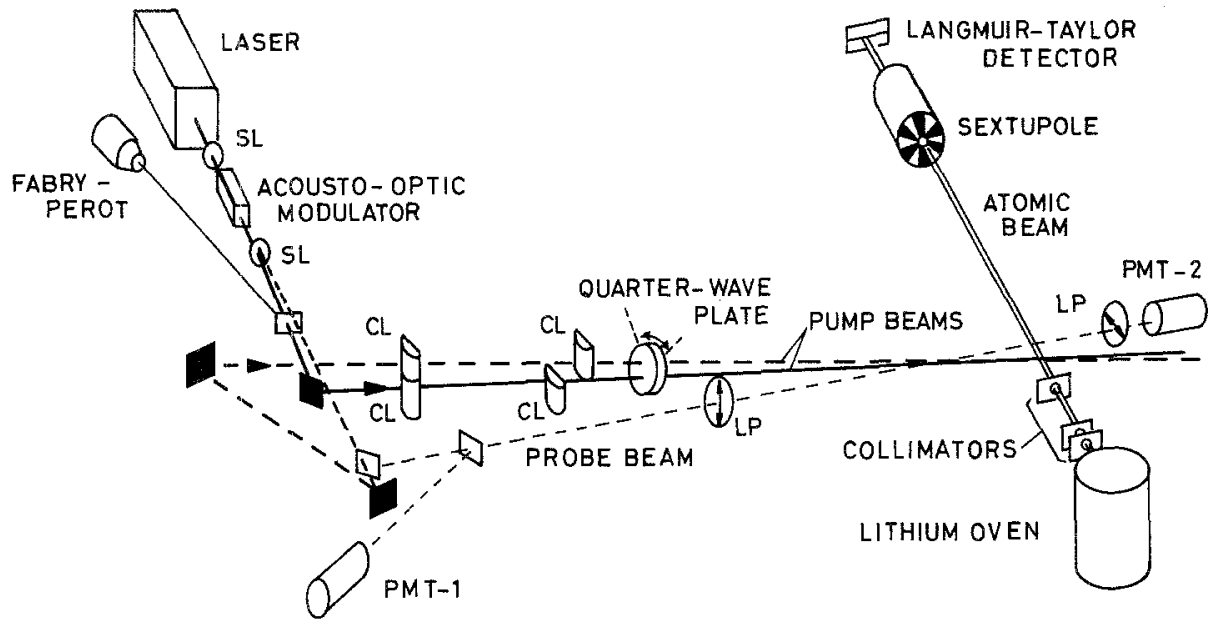

Fig. 2. Optical arrangement for focusing onto the lithium beam. The solid line is the frequency-unshifted light; the dashed line is frequency shifted by $228 \mathrm{MHz}$. The Fabry-Perot and PMT-1 are used for the stabilization of the intracavity laser etalon. Part of the frequency-shifted beam produces the crossed polarizer signal in PMT-2. Arrows indicate the direction of linear polarization transmitted by the linear polarizers (LP). Solid squares are mirrors; clear squares are beam splitters; SL denotes spherical lenses, and CL cylindrical lenses

estimate that the concentration of molecules in the beam is not more than $0.75 \%$ of the total beam intensity.

Our light source was a Spectra-Physics Model $580 \mathrm{~A}$ dye laser pumped with all lines of a Model $1654-\mathrm{W}$ $\mathrm{Ar}^{+}$laser. As dye we use a 1:1 mixture of Rh-101 and $\mathrm{Rh}-6 \mathrm{G}$. This gives a single mode output power of $20 \mathrm{~mW}$ at $6708 \AA$, the lithium resonance transition. The acousto-optic modulator (Soro IM50) was mounted outside the laser cavity. It was driven with an rf oscillator operating at $228 \mathrm{MHz}$. The unshifted laser beam passes through the center of the crystal and is tuned to the ${ }^{2} S_{1 / 2}, F=1 / 2 \rightarrow{ }^{2} P_{1 / 2}, F^{\prime}=1 / 2,3 / 2$ transition. The frequency-shifted beam pumps the other ground-state hyperfine component $(F=3 / 2)$.

Once the crystal has been optically aligned, the relative intensity in the two beams is controlled by the amount of power supplied to the transducer, and any ratio up to $1: 1$ (shifted: unshifted) can be achieved. For the pump process this is adequate. Our calculations, based on rate equations, show that as long as the intensity ratio between the frequency-shifted and frequencyunshifted beams is at least $1: 4$, we will achieve about the same degree of polarization as long as the more intense component is tuned to excite the lower hyperfine level.

The two light beams emerging from the crystal have the same beam profile but are separated in direction by the Bragg angle $\left(\sim 2^{\circ}\right)$. In order to utilize them properly for the pump process, Doppler shifting of one against the other must be avoided. This is most easily achieved by splitting the beams in a plane perpendicular to the direction of the lithium beam and crossing them at the atomic beam position. We apply a small magnetic field of several Gauss along the direction of the unshifted light beam, the quantization axis, in order to avoid effects due to stray magnetic fields.

Figure 2 shows a general diagram of the optics used to focus the laser beams onto the lithium beam. In addition to the mirrors required for alignment and the quarter-wave plate for producing the circular polarization, there are also two pairs of cylindrical lenses, one for each beam, for expanding the laser beams along the direction of the atomic beam. The ability to handle each beam separately is also especially useful in this respect, since we can broaden the frequency-unshifted beam slightly more than the shifted, thus obtaining a greater probability for removing atoms from the $F=1 / 2$ ground state.

As the polarized lithium source is intended for use in a scattering experiment, it is necessary that it remains stable over several hours. Since the principal cause of fluctuations in the polarization is frequency drift of the laser, we have designed a laser frequency stabilization circuit controlled by a microprocessor board (KIMI). The general idea behind this type of stabilization is due to Düren and Tischer [13]. We have extended their scheme to insure ourselves of a more precise control for the position of the intracavity etalon of the laser, thus optimizing the power output of the laser as well as stabilizing the frequency. We have also modified their scheme to our particular need of a stable polarization of the beam in that we control the laser frequency through direct feedback from the polarization of the beam itself rather than the maximum fluorescence of the lithium, which is not directly related to the maximum polarization of the beam [14].

In the stabilization program control of the frequency is performed in that the processor shifts the output frequency $v$ by a fixed amount $\Delta v$, first to a slightly 
lower, then to a slightly higher frequency and records at each position the beam polarization, $P(v)$. The same decrease in polarization in both directions indicates correct positioning of the output mirror of the laser; unequal changes or even increases indicate that a correction must be made. The etalon position is monitored constantly by a scanning Fabry-Perot interferometer, the position of the etalon being obtained from the time between the start of the scan and the appearance of the laser peak. The occurrence of mode hopping is recognized automatically and processor induced changes of the etalon position are made. Further, the laser intensity is monitored and a control analogous to the frequency control for the cavity allows continuous adjustment of the peak transmission of the etalon to sit atop the desired frequency. The microprocessor has also been programmed to finetune the laser to the lithium resonance transition and to rotate the quarter-wave plate.

\section{Polarization Analysis}

As polarization analyzer we employ a sextupole magnet following the scattering zone. The magnet has a length of $7.6 \mathrm{~cm}$ and a center bore of $3.2 \mathrm{~mm}$ diameter. In the strong magnetic field the Zeeman components are split according to the Breit-Rabi formula. Those components with spin parallel to the field (for lithium6 the states $F=3 / 2, M_{F}=-1 / 2,+1 / 2,+3 / 2$ ) are focused; components with spin antiparallel are defocused. The Langmuir-Taylor detector lies downstream from the sextupole, and a measurement of the polarization is accomplished as follows. First a recording is made of the signal without pumping, $I^{0}$. Then two measurements are made, the first with positive pumping, $I^{+}$, implying a population increase in the focused states, the second with negative pumping, $I^{-}$, implying a population decrease in the focused states. For a pure lithium- 6 beam the relative populations of the $(3 / 2,3 / 2),(3 / 2,1 / 2)$, and $(1 / 2 / 1 / 2)$ substates are proportional to the expressions $\left(I^{+}-I^{-}\right), I^{-}$, and $\left(2 I^{0}-I^{+}\right)$, respectively. It is assumed that the populations of the Zeeman sublevels with $M_{F}<0$ $\left(M_{F}>0\right)$ are equal to zero for $\sigma^{+}\left(\sigma^{-}\right)$pumping. Our rate equation calculations [14] indicate that this approximation is correct to within \pm 0.02 for spin polarizations exceeding 0.70 . We also assume that the pump process is entirely symmetric with respect to right and left pumping. Using the relative populations together with the electronic spin-polarization of the groundstate sublevels (Fig. 1) one then obtains the following formula for calculating the polarization of the lithium- 6 atoms in the beam:

$P_{\mathrm{Li}-6}=\left(\frac{\varepsilon^{+}-\varepsilon^{-}}{\varepsilon^{+}+\varepsilon^{-}}\right)^{-1} \times \frac{\left(I^{+}-I^{-}\right)+1 / 3\left(I^{-}+I^{+}-2 I^{0}\right)}{2 I^{0}(1-f)}(1)$
In this expression the factor $(1-f)$ corrects for that part of the signal which is due to lithium-7. For the specified isotopic content of the lithium used $f$ amounts to $f=0.05$. The quantities $\varepsilon^{+}$and $\varepsilon^{-}$are detection probabilities of the focused and defocused states, respectively. For the ideal analyzer the asymmetry factor in parentheses containing these constants will be equal to one. For the non-ideal analyzer it must be obtained on the basis of the velocity-dependent focusing characteristics of the sextupole. In our case it is estimated to be equal to $0.89 \pm 0.03^{3}$.

Important for the laser stabilization scheme as we have designed it is knowing the relative change of polarization as a function of such parameters as optical alignment, laser intensity, beam expansion, and frequency. This type of measurement can be accomplished quickly and sensitively. As a system which is spin polarized has a net magnetic moment, it exhibits different indices of refraction for the components of right $(+)$ and left $(-)$ circularly polarized light and is therefore birefringent. If linearly polarized light is passed through this system, the plane of polarization of the radiation will be rotated by an angle $\varphi$ proportional to the difference between these two indices, $n_{+}-n_{-}$. Analytic expressions for $n_{+}$and $n_{-}$for offresonant excitation have been calculated by Happer and Mathur [16]. These show that the net rotation of the plane of polarization is proportional to the sum of the magnetic moments of each individual hyperfine level, inversely weighted by the detuning from resonance. By selecting non-resonant light of slightly lower frequency than the ${ }^{2} S_{1 / 2}, \quad F=3 / 2 \rightarrow{ }^{2} P_{1 / 2}$, $F^{\prime}=1 / 2,3 / 2$ transition one is primarily sensitive to the magnetic moment of the $F=3 / 2$ state, and the rotation of the plane of polarization is directly correlated to the spin-polarization.

We measure this rotation of the plane of polarization in a region just following the pumping zone (Fig. 2). The incoming radiation is linearly polarized, and we analyze with a linear polarization analyzer following transmission through the lithium beam. These two polarizers are crossed, so as to give a zero signal in the photomultiplier when the lithium is unpolarized. As the system is pumped, however, rotation of the plane of polarization produces a signal through the second analyzer proportional to $\sin ^{2} \varphi$. As probing light we use a small portion of the frequency-shifted beam which is detuned from resonance by allowing it to intersect the atomic beam at an angle of approximately

\footnotetext{
3 Another possible means of measuring the polarization absolutely would be to analyze the system with polarized resonance radiation [15]. However, this method does not lend itself to fast, easily reproducible measurements of the polarization, which is what is required for the scattering experiment. Therefore, for our purpose, the sextupole result is quite adequate.
} 

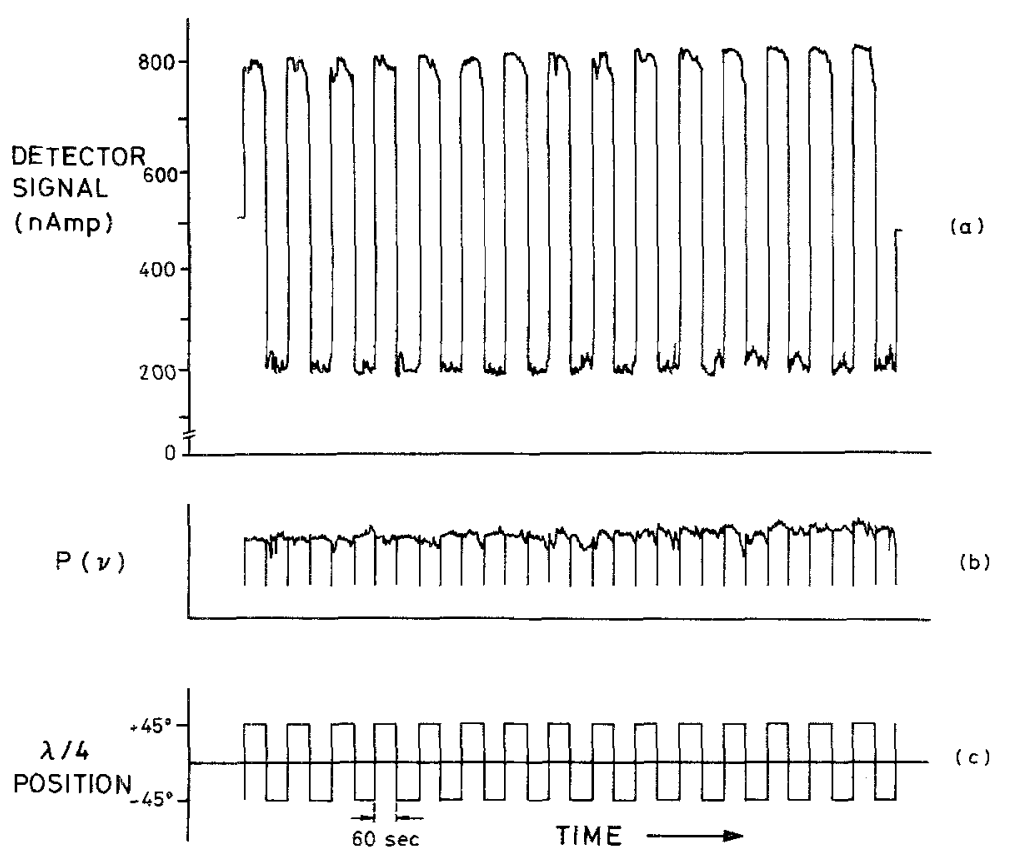

Fig. 3. (a) Intensity of lithium beam after passage through the sextupole as a function of quarter-wave plate position. (b) Signal from crossed polarizers as function of quarter-wave plate position. (c) Azimuthal position of principal axis of quarter-wave plate $2^{\circ}$. Since we only make relative measurements, we are not concerned that this method results in a velocityweighted signal. For our atomic polarization and a frequency detuning of about $100 \mathrm{MHz}$ from resonance, the angle $\varphi$ is about $2^{\circ}$. While we can measure this angle and the detuning from resonance quite accurately, we still do not know the lithium beam density well enough to be able to use this method to make absolute polarization measurements.

\section{Results}

Part (a) of Fig. 3 depicts the signal from the sextupole as a function of quarter-wave plate position, shown for comparison in part (c). The total beam intensity in this case is $1 \times 10^{14}$ atoms $^{-1}$. The laser power is $20 \mathrm{~mW}$, divided into a $1: 4$ ratio between the frequency-shifted and frequency-unshifted beams, respectively, and expanded over a length of $15 \mathrm{~mm}$ along the lithium beam. The pattern and stability of the signal are typical for operation of the polarized lithium atom source. The polarization of the lithium-6 atoms in the beam, calculated directly from the signals using (1), is $0.75 \pm 0.05$. The stated error is the uncertainty in determining the absolute polarization and is the combination of the uncertainties arising from the approximation made in deriving (1) and the errors made in estimating the constants $\varepsilon^{+}$and $\varepsilon^{-}$. The fluctuations of the polarization over a given period of time can be seen in the signal in Fig. 3. The small spikes are due largely to cavity-control steps in the laser stabilization program and contribute a fluctuation in the polarization of \pm 0.02 . The overall beam polarization, taking into account the unpolarized molecules and lithium-7 atoms, is calculated to be 0.70 .

While the scans in Fig. 3 were being recorded, the laser was stabilized to the maximum polarization of the beam, as measured by the crossed-polarizer signal. The sensitivity of this stabilization scheme can easily be seen from a comparison of this signal with that from the sextupole. Notice how accurately the spikes produced by the cavity-control steps in part (a) of Fig. 3 are reflected in a decrease of transmission through the second polarizer, part (b) of Fig. 3. Since three successive measurements are required to measure the spin polarization with the sextupole, it would be impossible to achieve this sensitivity with the sextupole alone. Also note that the crossed-polarizer signal confirms the symmetry of the pump process with respect to right and left pumping. Whether the atoms are pumped into the $F=3 / 2, M_{F}=+3 / 2$ state $\left(I^{+}\right)$or into the $F=3 / 2$, $M_{F}=-3 / 2$ state $\left(I^{-}\right)$, the crossed-polarizer signal is the same. This is a valuable check on the adjustment of the quarter-wave plate at $\pm 45^{\circ}$.

Based on a beam velocity of $2000 \mathrm{~m} / \mathrm{s}$ and a collimation ratio of 100 we calculate a Doppler width of $25 \mathrm{MHz}$ for a pumping zone extending over the entire beam, which is reduced to $10 \mathrm{MHz}$ for the central portion of the beam used for scattering and mainly analyzed by the sextupole magnet. Thus the hfs-splitting of the $2 P_{1 / 2}$ state $(26.2 \mathrm{MHz})$ is partially resolved in the Langmuir-Taylor signal, but is not resolved in the fluorescent light signal. These observations place an upper limit of about $10 \mathrm{MHz}$ on the laser bandwidth. The natural width of the $2 s-2 p$ transition is $6 \mathrm{MHz}$. 
The experimental result for the lithium- 6 beam polarization is in agreement with our rate equation calculations which give a value of $P_{\mathrm{Li}-6}=0.73$ for the parameters stated above, assuming pumping over the $F^{\prime}=3 / 2$ sublevel. As the calculations either assume that the hfs-splitting of the excited state is not resolved or resolved completely, they approximate the experimental situation which shows comparable size for Doppler width, hfs-splitting, and laser bandwidth. For a more precise calculation these finer details would have to be taken into account and, in addition, effects of unequal beam expansion for the frequency-shifted and unshifted pump beam.

Our measurements indicate that with the present laser flux density of $30 \mathrm{~mW} / \mathrm{cm}^{2}$ the transition is saturated. In order to increase the atomic beam polarization the number of spontaneous decay transitions which an atom experiences in the pumping region has to be increased. This can be done by increasing the light beam expansion along the atomic beam direction. The rate equations indicate that going from the present $15 \mathrm{~mm}$ expansion to $20 \mathrm{~mm}$ and running at a slightly more favourable intensity ratio for the two pump beams of $1: 1$ will lead to a predicted polarization of 0.85 . While the scattering experiment is being performed attempts in this direction will be undertaken as well as steps for allowing a more precise determination of the polarization with the sextupole magnet.

It is noteworthy that, in addition to the high electronic polarization, the lithium-6 atomic beam likewise possesses a high nuclear polarization. Through the optical pumping the state $F=3 / 2, M_{F}=+3 / 2\left(M_{F}=-3 / 2\right)$ is primarily occupied, and this means that most of the nuclei have $M_{I}=+1\left(M_{I}=-1\right)$, equivalent to a large nuclear vector polarization.

\section{Conclusions}

It is shown that the method of optical pumping with resonant laser light can be used to spin-polarize an alkali atomic beam. The method produces high polarization in low magnetic fields. It also allows for easy reversal of the direction of polarization without large systematic errors, thus overcoming the limitations connected with the more conventional method of polarization by state selection in a strong inhomogeneous magnetic field. A very convenient measure of the relative beam polarization is given by the birefringence of the polarized atomic beam for off-resonant light. This effect can be used efficiently to obtain a stable polarization over long terms.

Acknowledgements. We would like to thank Professor W. Raith for many helpful discussions. This research has been supported by the University of Bielefeld under Project No. 2845.

\section{References}

1. H. Kleinpoppen: Phys. Rev. A3, 2015-2027 (1971)

2. P.F.Wainwright, M.J.Alguard, G.Baum, M.S.Lubell : Rev. Sci. Instrum, 49, 571-585 (1978)

3. W.Raith, G.Baum, C.D.Caldwell, E.Kisker: In Physics of Atoms and Molecules, Proc. Intern. Workshop on Coherence and Correlation in Atomic Collisions (Plenum Press, New York, London 1979) pp. 567-575

4. W.Happer: Rev. Mod. Phys. 44, 169-249 (1972)

5. H.S.Pilloff: Appl. Phys. Lett. 21, 339-340 (1972)

6. I.V.Hertel, A.S.Stamatović: IEEE J. Quant. Elect. QE-11, 210-212 (1975)

7. F.Schuda, C.R.Stroud, Jr. : Opt. Commun. 9, 14-16 (1973)

8. H.J.Gerritsen, G. Nienhuis : Appl. Phys. Lett. 26, 347-349 (1975)

9. G.M.Carter, D.E.Pritchard, T.W.Ducas: Appl. Phys. Lett. 27, 498-499 (1975)

10. E.I.Gordon: Appl. Opt. 5, 1629-1639 (1966)

11. W.Franzen, A.G.Emslie: Phys. Rev. 108, 1453-1458 (1957)

12. M.J.Alguard et a1.: Nucl. Instrum. Methods 163, 29-59 (1979)

13. R. Düren, H. Tischer: Max-Planck-Institut für Strömungsforschung Göttingen, Bericht 4 (1978)

14. W.Schröder: Diplomarbeit, University of Bielefeld (1979) unpublished

15. A.Fischer: Diplomarbeit, University of Kaiserslautern (1978) unpublished

16. W.Happer, B.S.Mathur: Phys. Rev. Lett. 18, 577-580 (1967) 